\title{
Predicting the compaction of hybrid multilayer woven composite reinforcement stacks
}

M. Valkovaa, b, D. B. Anthonya, b, c, A. R. J. Kucernakc, M. S. P. Shaffer ${ }^{b, c, d}$, and E. S. Greenhalgha, b, *

a Department of Aeronautics, Imperial College London, South Kensington Campus, London SW7 2AZ, United Kingdom

b The Composites Centre, Imperial College London, South Kensington Campus, London SW7 2AZ, United Kingdom

c Department of Chemistry, Imperial College London, South Kensington Campus, London SW7 2AZ, United Kingdom

a Department of Materials, Imperial College London, South Kensington Campus, London SW7 2AZ, United Kingdom

Email Addresses:

maria.valkova13@imperial.ac.uk; d.anthony08@imperial.ac.uk; anthony@imperial.ac.uk; m.shaffer@imperial.ac.uk, e.greenhalgh@imperial.ac.uk

"Corresponding author: e.greenhalgh@imperial.ac.uk (E. S. Greenhalgh)

\section{Abstract}

A meso-scale modelling strategy was developed to investigate the effect of hybridisation on the compaction response of multilayer stacks combining different dry woven fabrics. It is expected that the electrochemical-mechanical properties of emerging multifunctional hybrid composites are strongly dictated by the morphology of the compacted reinforcements, yet no investigations into their compressibility have been reported. Finite element unit cell models were constructed from the average geometric and compressibility properties of the constituent glass and carbon fabrics, considering different stacking sequences and ply offsets. Model predictions were evaluated against compressibility measurements for monolithic and hybrid fabric stacks. The ply offset was found to have a major influence on the predicted internal morphologies and fibre content, contributing to experimental variability thereof. Optical microscopy and micro X-ray computed tomography imaging further indicated greater likelihood of intermediate ply offsets in physical specimens, over limit case idealisations. Compressibility was slightly reduced in the hybrid multilayer stacks studied in this work. The model outputs presented are being used to analyse the electrochemical-mechanical response of monolithic and hybrid woven structural power composites.

Keywords: A. Fabrics/textiles; A. Hybrid; C. Process Simulation; E. Consolidation 


\section{Introduction}

Woven reinforcements are widely used in composite manufacture due to their handleability,

3

4

5

6

7

8

9

lower cost and improved formability over that of unidirectional prepregs [1]. Yarns can be woven into a variety of weave styles, permitting considerable control over the fabric forming characteristics, such as drapability, compressibility and permeability [1-4]. During the preforming and consolidation stages of manufacturing woven fabric-reinforced composites (WFRCs) the fabric architecture undergoes complex dimensional changes. The deformation mechanisms have been widely studied [5-8] and include changes in the local fibre orientation, volume fraction, and inter-yarn gaps, as well as yarn flattening and nesting between adjacent plies. Pressure is applied during consolidation to obtain composite parts with high fibre content and minimal defects. The structural integrity of the resulting WFRC component is strongly influenced by its micro- and meso-structure, hence considerable efforts have been dedicated to their prediction through preforming and compaction process modelling.

Early semi-empirical and analytical models [9-11] of the compression of randomly-oriented and aligned fibre networks were based on fibre bending analysis, typically taking the form of a power law between applied pressure and fibre volume fraction. A meso-level finite element (FE) formulation for the compression of woven fabrics was later proposed by Lomov and Verpoest [12], considering a unit cell (UC) of the weave geometry and measured bending and compression properties of the yarns.

Subsequent FE models have highlighted the importance of lateral yarn interactions and material nonlinearity [13], and introduced more complex hypo-elastic [14,15] and hyper-elastic $[5,16]$ constitutive models.

Hybridisation of the laminate stacking sequence, by combining two or more fabrics with different weave styles, weights or fibre types, offers further opportunities to modify the properties of the resulting WFRCs. For example, hybrid woven fabric-reinforced composite (HWFRC) laminates containing carbon, glass and aramid fabrics have shown promise in improving impact and penetration performance[17-20]. Layering fabrics with different weave styles to create crimp gradients can enhance the damage tolerance of woven aramid composites [21]. Hybridisation of high performance synthetic fibre fabrics with natural fibre fabrics has been explored as a strategy for composite weight and cost saving and improved sustainability $[22,23]$. HWFRCs are also widely employed in the rapidly growing field of multifunctional structural power composites, such as structural batteries and structural supercapacitors [24-26], capable of storing/releasing electrical energy in addition to load bearing. 
Structural power composite 'cells' are typically fabricated as a three-layer stack consisting of carbon

2 fibre fabric outer plies (electrodes) and glass fibre fabric inner ply (separator), Thin glass fabric

3

4

5

6

7

8

9 separator plies provide a reasonable compromise between mechanical properties and electrochemical requirements, such as ensuring robust electrical insulation between the electrodes and minimising ionic resistance across the cell. Complex inter-ply nesting has been observed in these composites that is anticipated to have a significant influence on the ionic resistance across the cell, and hence the maximum achievable power density [27].

Though an area of intensive research, meso-scale fabric modelling has not yet investigated hybrid woven stacking sequences. Multilayer compaction models $[15,16,28]$ have been restricted to mono-fabric (hereafter referred to as monolithic) stacks only, perhaps due to the difficulty in applying the UC modelling technique, on which meso-scale modelling is typically reliant on, to more complex stacking sequences. A lack of simulation tools has hindered investigations of the effects of WFRC hybridisation in both conventional structural and multifunctional applications. Modelling the compaction of hybrid woven stacks could additionally provide insight into the effect of hybridisation on compressibility, highlighting any manufacturing considerations. Finally, such compaction and forming models could serve as a pre-processing tool in the generation of composite finite element models with more realistic descriptions of the local yarn geometry, interactions and fibre volume fraction distributions in HWFRCs.

In this work, a new approach for modelling hybrid woven reinforcement stacks, combining carbon and glass fabrics is proposed, motivated by their application in electrochemical structural power composites developed by our Group [24,25]. Firstly, the compressibility characteristics of a glass and a carbon fibre dry fabric are characterised in an out-of-plane compression experiment. A UC finite element model of each fabric ply is constructed, in which the yarn constitutive behaviour is governed by a non-linear relation calibrated using the experimental data. The resulting constitutive relations are subsequently used to simulate the compaction behaviours of monolithic bilayer stacks, as well as hybrid bi- and trilayer stacks combining the two fabrics. A hybrid unit cell (hUC) is proposed to model the latter. The multilayer model predictions are assessed against experimental compressibility data, as well as optical microscopy and X-ray micro-computed tomography $(\mu \mathrm{CT})$ scans of a hybrid composite laminate. The model is intended to serve as a virtual compaction test tool 
for hybrid woven stacks, providing valuable information about the attainable fibre volume fractions and

2 morphologies.

\section{2. Experimental procedures}

\section{$4 \quad 2.1 \quad$ Mechanical characterisation of fabric compressibility}

Two fabrics were used in this study: a $200 \mathrm{~g} / \mathrm{m}^{2}$ conventional carbon fibre plain weave from Chomarat (C-WEAVE ${ }^{\mathrm{TM}}$ 200P $3 \mathrm{~K}$ HS) [29] and a $53 \mathrm{~g} / \mathrm{m}^{2}$ spread tow glass fibre plain weave from Gividi Fabrics srl (1086) [30]. This choice of materials was motivated by their use as electrode and separator fabrics in structural supercapacitors investigated by the group [31]. Single plies (monolayers) of these carbon and glass fabrics are hereafter referred to by codes $\mathrm{C}$ and $\mathrm{G}$, respectively. Surface scans of the fabrics are shown in Fig. 1 (a) \& (b) and the fabric specifications are presented in Table 1.

The compressibility experiments were performed by applying out-of-plane compressive load to fabric specimens via parallel platens. Parallelism between the platens was ensured by using a twopart self-aligning spherical joint compression fixture of $50 \mathrm{~mm}$ diameter (Fig. 1 (c)). Specimens with dimensions $60 \times 60 \mathrm{~mm}$ were cut from the fabrics and placed centrally on the fixture. In addition to the monolayer specimens, $C$ and $G$, the compressibility behaviour of multilayer specimens with the following stacking sequences were studied: $\mathrm{C}_{2}, \mathrm{G}_{2}, \mathrm{CG}, \mathrm{GCG}$ and CGC. The hybrid CGC stacking sequence was of particular interest in this work, since it reflects the architecture of electrochemical structural power composites. All specimens were inspected before testing and care was taken to ensure good alignment of the fibre directions. However it is impossible to eliminate all misalignments; some misalignment (ca. $<2^{\circ}$, estimated from the minimum angle of misalignment resolvable by the naked eye) was likely still present. The specimens were loaded up to a pressure of $1 \mathrm{MPa}$, corresponding to an applied force of about $2 \mathrm{kN}$, and immediately unloaded by returning the crosshead to its initial position. No preload was used. The test was performed on an Instron 5969 universal testing machine fitted with a $50 \mathrm{kN}$ load cell, calibrated within $-0.25 \%$ error in compression in the 0.5 to $2 \mathrm{kN}$ force range. The experiments were performed in displacement-control at a rate of 1 $\mathrm{mm} / \mathrm{min}$. The specimens were loaded only once, and the first compression cycle was taken to represent realistic forming conditions. Irreversibility during subsequent loading-unloading cycles was therefore not considered in this work. At least six specimens were tested per stacking sequence. 
2 Gauge system. A General Purpose Lens (ISM-LENS-GP006, $50 \mathrm{~mm}$ focal length, $0.12 \mu \mathrm{m}$ typical

3 displacement resolution, with 80 pixels equal to $2.0 \mathrm{~mm}$ ) or a Material Test Lens (IMT-LENS-MT051,

40.394 magnification, $190 \mathrm{~mm}$ working distance, $0.070 \mu \mathrm{m}$ typical displacement resolution, with 80

5 pixels equal to $1.1 \mathrm{~mm}$ ), both from Imetrum Ltd, was fitted to the camera (IMT-CAM027) depending on the total thickness of the specimen. This optical measurement method eliminated the need for any additional machine compliance calibrations.

The calculation of the total fibre volume fraction at a given applied pressure, $V_{f}^{\text {total }}(p)$, was adapted from [15] for hybrid fabric stacks,

$$
V_{f}^{\text {total }}(p)=\frac{1}{t(p)}\left(\frac{n_{\mathrm{C}} m_{s, \mathrm{C}}}{\rho_{\mathrm{C}}}+\frac{n_{\mathrm{G}} m_{s, \mathrm{G}}}{\rho_{\mathrm{G}}}\right),
$$

where $t(p)$ is the specimen thickness (i.e. vertical distance between the platens) at the applied pressure $p, n$ is the number of fabric layers, $m_{s}$ is the fabric areal mass, $\rho$ is the density of fibres and additional subscripts $\mathrm{C}$ and $\mathrm{G}$ refer to the respective fibre type.

\section{$2.2 \quad$ Imaging techniques}

Optical microscopy was used for the geometric characterisation of $C$ and $G$ in their undeformed state. The samples were prepared by casting pieces cut from the fabric in polyester mounting resin/hardener system, taking care to align the fabric orientation with the through thickness sample direction, and polishing the surface after cure. Cross-sections with approximate widths of $20 \mathrm{~mm}$ were prepared along both the warp and weft directions for each fabric and imaged in a Zeiss Axio Imager.M2m optical microscope.

Both optical microscopy and $\mu C T$ were used to qualitatively validate the geometry of the CGC model. A CGC composite panel was manufactured by epoxy resin infusion under flexible tooling. The epoxy resin contained bisphenol A diglycidyl ether monomer with isophorone diamine hardener, and panels were cured according to the manufacturer's instructions. A diamond wire saw was used to cut samples from the cured panel. To produce the optical microscopy specimen, five samples with approximate in-plane dimensions of $20 \times 20 \mathrm{~mm}$ were cut from the panel and cast in mounting resin. The specimen cross-section was then polished and observed at 20x magnification. The $\mu C T$ specimen was produced from three samples cut from the panel, each with approximate in-plane (xy) dimensions of $10 \times 10 \mathrm{~mm}$, stacked in the through-thickness $(\mathrm{z})$ direction and secured with tape. The 
scan was performed at the National Composites Centre, GB using a Nikon X-Tek 320 kV electron beam machine. The beam voltage and current used were $60 \mathrm{kV}$ and $103 \mu \mathrm{A}$. The voxel size was $6.065 \mu \mathrm{m}$ with 3141 radiographs acquired during one full rotation of the specimen on the rotation stage. The Metris CT Pro software was used to generate a volume reconstruction from the radiographs using a filtered back-projection reconstruction algorithm. Additional manipulation, segmentation and visualisation of the scanned volume was performed in VG Studio Max 2.1 and Avizo 9. The scan results are discussed in Section 5.2.

\section{Monolayer fabric model}

\subsection{Geometric model}

In meso-scale fabric modelling, the individual fibres and their interactions are not modelled discretely; instead, the fabric architecture is represented by the yarns. The yarns are treated as solid continua with homogenised material properties that are isotropic in the plane of the yarn crosssection. It should be noted that the resulting stress-strain fields within the yarn should not be interpreted as those at the micro-scale. It is assumed that the yarns' local shape, spacing, contact interactions, fibre orientation and fibre volume fraction are sufficient to represent the mechanical and kinematic behaviour of the fabric.

The above approach was adopted in the present work. Simplifying assumptions were employed, allowing the geometric unit cell model of a fabric monolayer to be constructed from three basic parameters: yarn width, pitch and thickness. Average values of these parameters in the undisturbed state were determined by optical microscopy (Fig 2 (a) \& (b)) and are summarised in Table 2.

The assumptions were, specifically, that (i) the weaves were nominally balanced, i.e. identical geometric and material properties of the warp and weft yarns, ignoring any potential variabilities introduced by the weaving process [13]; (ii) yarn cross-sections were approximately elliptical in the absence of contact interactions between neighbouring yarns [32]; (iii) the yarns followed an approximately sinusoidal path in undeformed plain weave fabrics [33].

The geometry was generated in Abaqus/CAE through the Abaqus scripting interface (Fig 2 (c) \& (d)). Idealised yarn volumes were constructed by spline extrusions of the cross-section along the yarn paths. A common problem of the above approach is that, except when applied to very sparse fabrics, the resulting idealised geometric models contain undesirable volumetric interpenetrations [34]. 
In this work, the interpenetrations were resolved using the fully-automated procedure outlined in [35], consisting of a fictitious thermal contraction-expansion step with activated contact interactions between the yarns during expansion. The latter introduced a more realistic spatial variability in the yarn cross-section at no additional computational cost.

\subsection{Domain discretisation and boundary conditions}

A structured hexahedral mesh was constructed by partitioning the yarn volumes such that the elements preserved the local longitudinal and transverse directions of the yarn. The automatic periodicity of the resulting mesh facilitated the application of in-plane periodic boundary conditions through Multi-Point Constraint equations [36]. In these equations, the nodal displacement degrees of freedom of pairs of equivalent nodes on opposite thorough-thickness faces of the unit cell were linked through those of a fictitious master node. Out-of-plane compressive load was simulated using a coupling constraint, linking the out-of-plane displacement degrees of freedom of a pair of steel platens on either side of the fabric UC to those of an additional master node.

The fabric deformation was modelled in the large displacement framework (geometrically nonlinear analysis). Contacts were modelled using a global surface-to-surface contact algorithm. A 'hard' contact definition was specified for the normal contact behaviour. Tangential contact behaviour was described by a global penalty friction formulation with an assumed inter-yarn friction coefficient of 0.2 for $C[37,38]$, and 0.5 for $G[13,39]$. Convergence difficulties due to contact non-linearity are common to this type of problem $[40,41]$, and in the present case necessitated the use of the Abaqus/Explicit dynamic solver. Care was taken to ensure that all analyses were performed in a quasi-static regime by controlling the rate of applied displacement and mass scaling, and monitoring the relevant strain and kinetic energy outputs.

First order hexagonal reduced integration elements (C3D8R) were used in this work, after demonstrating better accuracy than alternative hexagonal element formulations in preliminary comparative studies. Care was taken to produce high-quality approximately rectangular elements, however, some element skewing at the yarn edges was unavoidable due to the elliptical yarn crosssection. It must be highlighted that parallelogram- and trapezium-shaped elements with skew angles as low as $10^{\circ}$ suffer substantial loss of accuracy [42], with significant implications for the accuracy of fabric forming simulations. In the present work, a very fine mesh was used to minimise such skew. 
1 Enhanced hourglass control was used and the artificial energy dissipation of the model was

2 monitored.

\section{$3 \quad 3.3 \quad$ Constitutive model}

4

Achieving consistency between the mechanical behaviour of a fabric meso-scale model with that observed in experiments is a challenging task. Owing to the weaving of the yarns, dry fabrics exhibit a quasi-continuous behaviour [43] at both the yarn and fabric scales, despite discontinuity at the fibre scale. It is widely accepted that yarns should be modelled as transversely isotropic [28], and that their fibrous structure gives rise to a number of unique mechanical characteristics: the yarn tensile and bending stiffnesses are decoupled, and the transverse, bending and shear stiffnesses are small in comparison to the longitudinal tensile stiffness [14]. In addition to geometric non-linearity and evolving contact boundary conditions between yarn and tool surfaces, fabric-forming simulations should consider the material non-linearity associated with the changing fibre packing density.

With the exception of the transverse modulus, the elastic properties of the yarns used in this work were adopted from literature (Table 3). A non-linear relationship between the local (intra-yarn) fibre volume fraction, $V_{f}$, and transverse modulus, $E_{2}$, was implemented through a user-defined material subroutine (VUMAT). The element-wise intra-yarn fibre volume fraction was introduced as a solution dependent variable and calculated based on the initial intra-yarn fibre volume fraction, $V_{f 0}$, and the element volume change, which is represented by the element Jacobian, $J$ :

$$
V_{f}=\frac{V_{f 0}}{J} .
$$

The Jacobian was obtained from the deformation gradient, $F$, as $J=\operatorname{det}(F)$.

The initial fibre volume fraction (Table 2) was computed from the fabric areal mass, density of fibres, unit cell side length, $L$, and idealised yarn volume contained within the unit cell , $V_{y a r n s, 0}$, according to:

$$
V_{f 0}=\frac{m_{s} L^{2}}{\rho V_{\text {yarns }, 0}} .
$$

Adopting the methodology used in previous works $[12,15,16]$, the non-linear transverse modulus of the yarns was assumed to follow a bi-exponential relation

$$
E_{2}=a \exp \left(b V_{f}\right)+c \exp \left(d V_{f}\right),
$$

where $E_{2}$ is measured in $\mathrm{Pa}$, while $a, b, c$ and $d$ are fitting coefficients. The coefficients $a$ and $b$ control the low pressure response, while $c$ and $d$ control the high pressure response. An iterative inverse 
procedure was used to obtain the values of the coefficients (Table 4) by minimising the error between the experimental and numerical compressibility responses for each of the two fabric monolayers. A mesh convergence analysis performed on the fabric monolayer models (Fig. 3) indicated that in both cases solution convergence was achieved with 2142 elements per yarn.

\section{Multilayer fabric model}

\subsection{Monolithic unit cell}

Models of the bilayer monolithic stacks $\mathrm{C}_{2}$ and $\mathrm{G}_{2}$ were generated to enable comparison between the compaction behaviours of monolithic and hybrid stacking sequences, and to provide additional model validation datasets. Inter-ply nesting is a key feature of multilayer fabric stacks and has a significant influence on compaction behaviour [7]. The relative in-plane ply offset of the fabric plies determines the size and position of the yarn contact regions, through which compressive load transfer occurs, and consequently the effective compressibility of the stack. The concept of phase difference between the idealised sinusoidal yarn paths was adopted to describe the ply offset (nesting configuration) [15]. To account for the potential range of effective compressibilities, the limit cases representing minimum and maximum possible nesting were considered: in-phase (IP) and $90^{\circ}$ out-ofphase $\left(90^{\circ} \mathrm{OP}\right)$. The two cases can alternatively be described as no in-plane offset between adjacent fabric plies, and an offset equal to $25 \%$ of the UC length, respectively (Fig. 4). The alignment of the contact points (i.e. yarn densification regions) in the IP model is expected to yield a compressibility response equivalent to that of the monolayer. In the $90^{\circ} \mathrm{OP}$ model, the multitude and spread of contact regions across the unit cell is expected to result in more uniform yarn densification and higher total fibre volume fraction at a given applied pressure, relative to the IP model and monolayer cases."

This methodology enabled comparison with the experimentally-determined compressibility ranges, where a random variation of ply offsets are present. The constitutive models of the $C$ and $G$ yarns were those obtained from the previously described initial calibration performed on the monolayer models (Section 3.3), while boundary conditions and remaining model parameters were kept the same. Additionally, the equivalence of the in-phase model to a $180^{\circ}$ out-of-phase model [44] was verified by comparison between their respective compressive responses. 


\subsection{Hybrid unit cell}

The major challenge associated with constructing a unit cell model of a hybrid woven reinforcement stack, or hUC model, stems from the dissimilar unit cell dimensions of the constituent fabrics. Consider the unit cells of two balanced fabrics $A$ and $B, \cup C_{A}$ and $U C_{B}$, with unique side lengths, $L_{\mathrm{A}} \neq L_{\mathrm{B}}$. To construct a meso-scale hUC of a multilayer stack consisting of the two fabrics periodicity of the hUC geometry must be ensured. Strictly speaking, a periodic hUC can only be generated by the tessellation of $U C_{A}$ and $U C_{B}$, such that the resultant side length of the $\mathrm{hUC}, L_{\mathrm{hUC}}$, represents the least common multiple of those of the constituent unit cells, i.e. $n_{\mathrm{A}} L_{\mathrm{A}}=n_{\mathrm{B}} L_{\mathrm{B}}$, where $n_{\mathrm{A}}, n_{\mathrm{B}} \in \mathbb{N}$. The number of required tessellations is then $n_{\mathrm{A}}^{2}+n_{\mathrm{B}}^{2}$ for a hybrid bilayer. In most cases, strict enforcement of this requirement would render the size of the hUC mesh impractical for mesoscale FE analysis. It is therefore desirable to make suitable approximations where possible to reduce the computational domain.

The approach proposed, here, is to approximate the in-plane geometric parameters of the fabrics to minimise the number of tessellations required for a periodic hUC. First, values of $L_{A}$ and $L_{B}$ can be modified within the range of specimen variability (mean \pm one standard deviation), such that their least common multiple, and hence $n_{A}$ and $n_{B}$ are minimised. To satisfy the modified UC length, yarn width and pitch can be adjusted by selecting appropriate values from their respective ranges of measurement variability, as close as possible to the mean. The intra-yarn fibre volume fraction can also be adjusted to ensure the total fibre volume fraction of the modified UC matches that of the original UC. In this work, the absolute differences in the initial intra-yarn volume fraction between the modified and the original geometries were $-0.66 \%$ and $+0.62 \%$ for the $\mathrm{C}$ and $\mathrm{G}$ fabrics respectively, hence no additional volume fraction adjustment was deemed necessary.

Using the modified geometric parameters (Table 5) allowed hUCs containing fabrics $\mathrm{C}$ and $\mathrm{G}$ to be constructed with a 1:5 tessellation ratio (Fig. 5). IP and $90^{\circ} \mathrm{OP}$ nesting of the $\mathrm{C}$ layers were considered in the CGC stacking sequence. Additional nesting configurations were not considered for the remaining stacking sequences CG and GCG due to the fineness of the G plies (further discussed in Section 6.1). 


\subsection{Compressibility response comparison}

The experimental curves of total fibre volume fraction against applied pressure (Fig. 3 and Fig. 6) are presented as an experimental range, calculated from the mean plus or minus one standard deviation $( \pm 1 \sigma)$ for each stacking sequence, based on at least six specimens. The curves follow the same characteristic non-linear response demonstrated elsewhere in the literature $[13,15,16]$. The low initial compression resistance was attributed to a dominance of yarn bending and decrimping deformation mechanisms [7,45], while the sharp increase in compressive stiffness at higher applied compressive strains was attributed to increasing fibre packing density, and hence number of fibre-tofibre contact interactions within the yarns. The observed increase in compression resistance was approximately bi-exponential $\left(\mathrm{R}^{2}>0.98\right.$ in all cases), in the pressure range studied.

In the single layer experiments (Fig. 3) the initial fibre volume fractions of the fabric in their undeformed state were approximately $32 \%$ and $29 \%$ respectively for C and G. C presented a more rigid compression response $\left(V_{f, \mathrm{C}}^{\text {total }}(1 \mathrm{MPa})=49.5 \%\right)$ compared to $\mathrm{G}\left(V_{f, \mathrm{G}}^{\text {total }}(1 \mathrm{MPa})=55.3 \%\right)$. Both monolithic multilayer stacks, $\mathrm{C}_{2}$ and $\mathrm{G}_{2}$ (Fig. 6 (a)), showed increases in compressibility relative to the respective monolayer responses $\left(V_{f, \mathrm{C}_{2}}^{\text {total }}(1 \mathrm{MPa})=54.0 \%\right.$ and $\left.V_{f, \mathrm{G}_{2}}^{\text {total }}(1 \mathrm{MPa})=57.3 \%\right)$. This relative increase was attributed to the generation of nesting between the plies [15], and was more pronounced for $\mathrm{C}_{2}(8.7 \%)$, than $\mathrm{G}_{2}(3.6 \%)$. The measured compressibility curve ranges for the three hybrid stacking sequences (Fig. 6 (b)) fell between those of the monolayers C and G. Stacking sequence $\mathrm{CG}$, in which the mass fraction of $\mathrm{C}\left(m_{f, \mathrm{C}}\right)$ was $79 \%$, displayed the least compliant response $\left(V_{f, \mathrm{CG}}^{\text {total }}(1 \mathrm{MPa})=50.1 \%\right)$, dominated by the compressibility of $\mathrm{C}$. Sequence $\mathrm{GCG}\left(m_{f, \mathrm{C}}=\right.$ $88 \%)$ displayed an intermediate response $\left(V_{f, \mathrm{GCG}}^{\text {total }}(1 \mathrm{MPa})=51.9 \%\right)$, indicating an increased influence from the more compliant G plies. CGC was the most compliant on average $\left(V_{f, \mathrm{CGC}}^{\text {total }}(1 \mathrm{MPa})=52.4 \%\right)$. Significant experimental measurement variability was observed only for the $C_{2}$ and CGC stacking sequences, with $1 \sigma_{\mathrm{C}_{2}}(1 \mathrm{MPa})=2.2 \%$ and $1 \sigma_{\mathrm{CGC}}(1 \mathrm{MPa})=1.4 \%$, respectively. This variability was attributed to the range of possible nesting configurations of the $C$ plies, combined with their large initial thickness and crimp. All the other stacking sequences presented low scatter, with $1 \sigma<0.8 \%$. 

comparison (Fig. 6). As initially hypothesised, the experimental curves for the $\mathrm{C}_{2}, \mathrm{G}_{2}$ and CGC

3 stacking sequences fell inside the bounds provided by the respective IP and $90^{\circ} \mathrm{OP}$ model predictions. The latter offset configuration displayed the more compliant responses and more homogenous distributions of local fibre volume fraction (Fig. 7). The experimental ranges were far more restricted than the predicted bounding intervals, tending towards the IP limit condition. The compressibility responses of CG and GCG, however, were closely predicted.

\subsection{Meso-structure comparison}

From the $\mu \mathrm{CT}$ scan of the hybrid CGC/epoxy specimen, a volumetric reconstruction was obtained based on the local X-ray attenuation characteristics within each voxel (Fig. 8 (a)). High intensity voxels represent glass yarns, mid-intensity voxels represent carbon yarns and resin-rich regions, while the lowest intensities are associated with free air. Mutually orthogonal slices through the panel (Fig. 8 (b)) showed that the periodicity of the meso-structure was retained after compaction. Throughthickness waviness of the $G$ fabric was a key meso-structural feature. Although the morphology of the samples was relatively uniform, some local geometric variation, as well as shear distortion of the $G$ fabric (ca. $3^{\circ}$ ), were also present.

A $4.2 \times 4.2 \mathrm{~mm}$ region of interest containing one complete hUC was extracted from each of the three panel samples. Orthogonal slices (Fig. 9), particularly at the laminate midplane (xy), were used to qualitatively evaluate the accuracy of the compacted geometry predicted by the CGC models. Detailed statistical $\mu C T$ analysis was outside the scope the present modelling study; however, the reader is referred to literature for further information on its use in fabric characterisation $[46,47]$. To aid visualisation, the volume of a 'representative' hUC (Fig. 9 (b)) was segmented by defining threshold intensity values for the glass yarns and free air. Segmentation of the carbon yarns from pure epoxy regions was not possible due to the relatively low contrast between their respective grey values. Nevertheless, carbon yarns were still distinguishable by the orientations of carbon fibre bundles in the xy plane. The characteristic through thickness 'footprint' of the glass yarns at the laminate midplane slice (Fig. 10 (d)) was used to qualitatively assess the model meso-structure predictions.

Cross-sectional optical micrographs obtained from the same panel (Fig. 8 (c)) were used to complement the $\mu \mathrm{CT}$ observations, providing higher quality detail of the internal morphology. To aid 
visualisation and provide additional means of comparison, carbon yarns and glass yarns were segmented by tracing the yarn contours (Fig. 10 (h)).

Laminate midplane cuts (Fig. 10 (a) \& (b)) and cross-sections (Fig. 10 (e) \& (f)) extracted from the models showed that the compacted geometries from the two ply offset limit cases were in fact not closely representative of the physical specimen. These findings motivated the analysis of a third geometric configuration, $\mathrm{CGC}_{36^{\circ} \mathrm{OP}}$, with a phase shift of $36^{\circ}$ (a ply offset of $10 \%$ of the UC length) between the $\mathrm{C}$ plies in both the warp and weft directions, based on the average ply offsets observed in the physical $\mu \mathrm{CT}$ specimen (a total of 6 measurements, 2 per sample). The results of the $\mathrm{CGC}_{36^{\circ} \mathrm{OP}}$ model (Fig. 10 (c) \& (g)) yielded a much more accurate geometric representation of the panel.

\section{Discussion}

\subsection{Effect of hybridisation of the stacking sequence}

It is widely accepted that inter-ply nesting results in enhanced compressibility of monolithic multilayer stacks compared to that of single layers [15]. The experimental data presented in Fig. 3 \& Fig. 6 (a) further indicated that, for conventional woven fabrics, the scatter in compressibility was also greater in the multilayer case. The monolithic limit case FE model results (Fig. 6 (a)) demonstrated that this scatter was caused by variability in the ply offset. It was initially hypothesised that hybridisation of the stacking sequence would reduce the effective compressibility of the stack by inhibiting inter-ply nesting. Comparison between the compressibility curves of the $\mathrm{C}_{2,90^{\circ} \mathrm{OP}}$ and $\mathrm{CGC}_{90^{\circ} \mathrm{OP}}$ models (Fig. 6 (a) \& (b)) indicated that hybridisation of the stacking sequence slightly reduced the average total fibre volume fraction (ca. $2 \%$ at $1 \mathrm{MPa}$ ); meanwhile, the experimental data presented a similar reduction in the overall stack compressibility (1.6\% at $1 \mathrm{MPa})$, supporting this hypothesis. This relatively small reduction was initially surprising, however further analysis of the compressibility curves and the internal morphology of the physical specimen provided an explanation. The greater experimental scatter in the CGC response, compared to those in the remaining hybrid multilayer and the single layer specimens, suggested that some C-C nesting was present in the CGC specimens. Optical and $\mu$ CT observations (Fig. 8 (b), 9, 10 (d) \& (h)) confirmed that through-thickness waviness was introduced in the G ply, with a period and amplitude of the order of those of the $C$ plies. This characteristic microstructure was indicative of $\mathrm{C}-\mathrm{C}$ nesting, made possible by the relatively low areal weight and bending rigidity (improved conformability) of the $G$ fabric. 

sequences indicated that inter-ply nesting had a negligible influence on the compression response in those cases. In the former case, no matching fabric counter-surfaces were present in the layup, while in the latter, the matching counter-surfaces of the outer $G$ plies were separated by the thicker $C$ ply. These results lead to the conclusion that some loss of nesting, resulting from the loss of matching fabric counter-surfaces, may be expected in stacking sequences combining dissimilar weaves, however, this effect is specific to the stacking sequence and likely depends on the degree of geometric dissimilarity, the relative thicknesses and bending rigidities of the constituent fabrics. The choice of constituent fabrics may, therefore, influence the attainable fibre volume fractions and local architecture in the final HWFRC laminate. It is interesting to note that a similar loss of nesting was reported elsewhere in the case of monolithic fabric stacks containing off-axis plies [15], suggesting the impact of hybridisation may not be so apparent in laminates with quasi-isotropic layups.

\subsection{Modelling approach and practical variability}

The results in Section 5.1 confirm that the IP and $90^{\circ} \mathrm{OP}$ limit models of specimens $\mathrm{C}_{2}, \mathrm{G}_{2}$ and CGC predict the range within which the compressibility responses of the physical specimens lie, and the predictions compare well with those reported for monolithic twill fabric stacks [15]. The observed experimental scatter in compressibility is significantly narrower, however, suggesting that the limit case models represent conditions that are unlikely to occur in practice, while intermediate cases present a greater statistical likelihood. Furthermore, the experimental compressibility of these specimens tends towards the IP model predictions, where minimum nesting occurs. This tendency implies that physical multilayer specimens are subject to conditions which limit their compressibility, which were not considered in the models. These conditions may include i) global fabric pre-shear or relative misalignment of fabric plies $[8,15]$, ii) local misalignment of the yarn directions within plies [15], and iii) local dimensional deviation from the average parameters (variability) [48]. In each case, deviation from the assumed idealised geometry would prevent the occurrence of complete nesting as predicted at the $90^{\circ} \mathrm{OP}$ limit, and hence reduce the effective compressibility. This effect is expected to be especially significant for finer weaves, such as the fabric $G$ used in this study, since the geometric 'defect' count is expected to scale with the UC count per area; meanwhile, shear and distortion induced during handling is also more likely. The particularly restricted experimental compressibility range observed for the bilayer $\mathrm{G}_{2}$ appears to support the above statement. 

assumptions are of limited direct use. They provide a bounding interval of compressibility, since they assume a level of material uniformity and process control that is unrealistic. As evidenced by $\mu \mathrm{CT}$, some degree of geometric non-uniformity as well as pre-shear in the fabric G (Fig. 8 \& Fig. 10 (d)), was present even at the local scale of a few unit cells. Random ply shift UC models offer only a small improvement in this aspect, since they also contain an implicit assumption of global geometric uniformity.

The alternative is to consider a stochastic model, where geometric variabilities are randomly distributed across a much larger representative volume element [48], however, the computational cost associated with implementation in meso-scale non-voxel mesh models is prohibitive at present. Moreover, additional complications related to ensuring model periodicity [15] and robustness in models considering fabric pre-shear and yarn misalignment require further attention.

While the IP models do provide a closer approximation to the experimentally obtained compressibility curves than the $90^{\circ} \mathrm{OP}$ multilayer models, the resulting internal morphologies still do not correspond to those observed in physical WFRC and HWFRC specimens (Fig. 10). It is, therefore, more likely that misalignment and geometric variability are responsible for the low apparent compressibility of physical specimens, since a finite (non-zero) phase shift between the $\mathrm{C}$ plies was consistently observed in $\mu \mathrm{CT}$ and optical microscopy. The internal morphology of WFRCs is highly sensitive to the local ply offset. It is consequently not surprising that, elsewhere, evidence from static compression tests [49,50] and static and fatigue delamination tests [51] suggests that the influence of nesting on the damage mechanisms and failure properties of monolithic WFRCs can be significant. Mechanical analyses performed on composite UCs and hUCs at the extreme as well as intermediate ply offset cases can provide insight into the distribution and sensitivity of local failure properties.

\subsection{Computational cost}

The mismatch in unit cell sizes in the hybrid system studied here, coupled with the demanding mesh size requirements for convergence, resulted in a very large FE mesh, easily exceeding 200,000 elements for a bilayer hybrid model with a 1:5 tessellation ratio. Furthermore, the numerous contact interactions necessitate the use of an explicit solution scheme, imposing time increment limitations. The solution of such a model is at present only feasible with the use of high-performance computing (HPC). Even with HPC, the computational cost would quickly become prohibitive for meso-models 
with less favourable tessellation ratios or larger stacking sequences. For this reason, the present study was limited to bi- and trilayer stacks. Out-of-plane symmetry or periodicity boundary conditions [35] could however be exploited to reduce the problem size for thicker stacks with suitable stacking sequences.

\section{Conclusions}

A method for meso-scale compaction modelling of dry multilayer reinforcement stacks with hybridised stacking sequences was proposed for the first time, which goes beyond existing mesomodels that have, thus far, been exclusively limited to monolithic stacks. The model was developed specifically for multi-weave stacking sequences combining different woven fabrics but is more widely applicable to any reinforcement architecture that can be described by a meso-scale unit cell (e.g. 3D woven, braided, non-crimp), including hybrid fibre reinforcements [52]. A domain minimization technique was employed for the generation of hybrid unit cell (hUC) models, which significantly reduces computational cost, and is a key contribution of this work. A simple non-linear constitutive model, calibrated using a single compression test per fabric and implemented within a user material subroutine, enabled tracking of the evolution of local fibre content during compaction.

The hUC models were used to study the kinematic and mechanical response of monolithic bilayer and hybrid bi- and trilayer fabric stacks during compaction, considering minimum, maximum and intermediate nesting cases. The combined experimental and numerical results demonstrated that the in-plane ply offset has a major influence on the compressibility responses and internal morphologies of both monolithic and hybrid multilayer stacks, while geometric non-uniformities and misalignments are likely further sources of variability. The results also indicated that hybridisation of the stacking sequence of woven fabric stacks may inhibit nesting, leading to a reduction in the attainable total fibre volume fractions compared to those of monolithic stacking sequences. The effect is expected to be dependent on the combination of weave types and bending stiffnesses of the constituent fabrics and future work plans to investigate this influence.

Finally, the model was developed as an automatic pre-processor for the generation of realistic representations of hybrid woven fabric-reinforced composites (HWFRCs), without the need for extensive geometric characterisation of physical specimens. Ongoing work uses compacted geometries obtained by the presented methodology to evaluate the electrochemical-mechanical performance of HWFRCs in structural power applications [24,25], however the methodology is more 
1 widely applicable to structural applications, where hybridised fabric stacking sequences receive

2 growing interest $[17,18]$. In contrast to dry fabric meso-modelling, where computational cost remains a 3 challenge, composite meso-models greatly benefit from the faster computation times associated with 4 implicit solution schemes. Considering the high sensitivity of the internal morphology to ply offset, its 5 influence on the failure properties of HWFRCs is a topic worthy of further research. 


\section{Acknowledgements}

This work has been funded by the EPSRC Future Composites Research Manufacturing Hub (EP/P006701/1), the EPSRC Beyond Structural project (EP/P007465/1), the European Office of Aerospace Research and Development (IOE Grant FA9550-17-1-0251) and EU Clean Sky 2 (SORCERER Project \#738085). The authors gratefully acknowledge Chomarat and Gividi Fabrics for supplying the materials used in this work. The authors also thank J. Meggyesi and K. Wolstenholme for their help with composites testing, and D. Ivanov and O. Nixon-Pearson for their help with acquiring the $\mu \mathrm{CT}$ scan and reconstruction.

\section{Conflicts of interest}

There are no conflicts to declare.

\section{Acronyms and symbols}

Applied pressure, $p$

Carbon, C

Deformation gradient, $F$

Density of fibres, $\rho$

Fabric areal mass, $m_{s}$

Fabric thickness, $t$

Fibre tensile modulus, $E_{f}$

Finite element, FE

Fitting coefficients, $a, b, c, d$

Glass, $\mathrm{G}$

High-performance computing, HPC

Hybrid unit cell, hUC

Hybrid woven fabric-reinforced composites, HWFRCs

Idealised yarn volume $V_{\text {yarns }, 0}$

In-phase, IP

Initial intra-yarn fibre volume fraction, $V_{f 0}$

Intra-yarn fibre volume fraction, $V_{f}$

Jacobian, $J$

Non-linear transverse modulus, $E_{2}$

Number of fabric layers, $n$

Out-of-phase, OP

Standard deviation, $\sigma$

Total fibre volume fraction, $V_{f}^{\text {total }}$

Unit cell, UC

Unit cell side length, $L$

Woven fabric-reinforced composites, WFRCs

$\mathrm{X}$-ray micro-computed tomography, $\mu \mathrm{CT}$ 


\section{References}

[1] Gereke T, Döbrich O, Hübner M, Cherif C. Experimental and computational composite textile reinforcement forming: A review. Compos Part A Appl Sci Manuf 2013;46:1-10.

[2] Verleye B, Croce R, Griebel M, Klitz M, Lomov SV, Morren G, et al. Permeability of textile reinforcements: Simulation, influence of shear and validation. Compos Sci Technol 2008;68:2804-10.

[3] Rozant O, Bourban PE, Månson JAE. Drapability of dry textile fabrics for stampable thermoplastic preforms. Compos Part A Appl Sci Manuf 2000;31:1167-77.

[4] Bickerton S, Šimáček P, Guglielmi SE, Advani SG. Investigation of draping and its effects on the mold filling process during manufacturing of a compound curved composite part. Compos Part A Appl Sci Manuf 1997;28:801-16.

[5] Charmetant A, Vidal-Sallé E, Boisse P. Hyperelastic modelling for mesoscopic analyses of composite reinforcements. Compos Sci Technol 2011;71:1623-31.

[6] Mohammed U, Lekakou C, Dong L, Bader MG. Shear deformation and micromechanics of woven fabrics. Compos Part A Appl Sci Manuf 2000;31:299-308.

[7] Potluri P, Sagar TV. Compaction modelling of textile preforms for composite structures. Compos Struct 2008;86:177-85.

[8] Ivanov DS, Lomov SV. Compaction behaviour of dense sheared woven preforms: Experimental observations and analytical predictions. Compos Part A Appl Sci Manuf 2014;64:167-76.

[9] van Wyk CM. 20-Note on the compressibility of wool. J Text Inst Trans 1946;37:T285-92.

[10] Gutowski TG, Dillon G. The elastic deformation of lubricated carbon fiber bundles: Comparison of theory and experiments. J Compos Mater 1992;26:2330-47.

[11] Manson JE. Elastic compression of a fiber network. J Appl Mech 1995;62:1993-6.

[12] Lomov SV, Verpoest I. Compression of woven reinforcement: a mathematical model. J Reinf Plast Compos 2000;19:1329--1350.

[13] Lin H, Sherburn M, Crookston J, Long AC, Clifford MJ, Jones IA. Finite element modelling of fabric compression. Model Simul Mater Sci Eng 2008;16.

[14] Gasser A, Boisse P, Hanklar S. Mechanical behaviour of dry fabric reinforcements. 3D simulations versus biaxial tests. Comput Mater Sci 2000;17:7-20.

[15] Nguyen QT, Vidal-Sallé E, Boisse P, Park CH, Saouab A, Bréard J, et al. Mesoscopic scale analyses of textile composite reinforcement compaction. Compos Part B Eng 2013;44:231-41.

[16] Thompson AJ, El Said B, Ivanov D, Belnoue JPH, Hallett SR. High fidelity modelling of the compression behaviour of 2D woven fabrics. Int J Solids Struct 2017.

[17] Chen D, Luo Q, Meng M, Sun G. Low velocity impact behavior of interlayer hybrid composite laminates with carbon/glass/basalt fibres. Compos Part B Eng 2019;176:107191.

[18] Cheon J, Lee M, Kim M. Study on the stab resistance mechanism and performance of the carbon, glass and aramid fiber reinforced polymer and hybrid composites. Compos Struct 2020;234:111690.

[19] Enfedaque A, Molina-Aldareguía JM, Gálvez F, González C, Llorca J. Effect of glass fiber hybridization on the behavior under impact of woven carbon fiber/epoxy laminates. J Compos Mater 2010;44:3051-68.

[20] Sevkat E, Liaw B, Delale F, Raju BB. Drop-weight impact of plain-woven hybrid glassgraphite/toughened epoxy composites. Compos Part A Appl Sci Manuf 2009;40:1090-110.

[21] Cavallaro P V. Effects of weave styles and crimp gradients in woven kevlar / epoxy composites 2016:617-35.

[22] Yahaya R, Sapuan SM, Jawaid M, Leman Z, Zainudin ES. Effect of layering sequence and chemical treatment on the mechanical properties of woven kenaf-aramid hybrid laminated composites. Mater Des 2015;67:173-9.

[23] Gujjala R, Ojha S, Acharya SK, Pal SK. Mechanical properties of woven jute-glass hybridreinforced epoxy composite. J Compos Mater 2014;48:3445-55.

[24] Greenhalgh ES, Ankersen J, Asp LE, Bismarck A, Fontana QPV, Houlle M, et al. Mechanical, electrical and microstructural characterisation of multifunctional structural power composites. $J$ Compos Mater 2015;49:1823-34.

[25] Shirshova N, Qian H, Houlí M, Steinke JHG, Kucernak ARJ, Fontana QPV, et al. Multifunctional structural energy storage composite supercapacitors. Faraday Discuss 2014;172:81-103.

[26] Hudak NS, Schlichting AD, Eisenbeiser K. Structural Supercapacitors with Enhanced 
Performance Using Carbon Nanotubes and Polyaniline. J Electrochem Soc 2017;164:A691_ 700.

[27] Asp LE, Greenhalgh ES. Structural power composites. Compos Sci Technol 2014;101:41-61.

[28] Swery EE, Allen T, Kelly P. Predicting compaction-induced deformations of meso-scale textile models efficiently. J Compos Mater 2016:0021998316671572.

[29] Chomarat. Composites Reinforcements C-weave ${ }^{\mathrm{TM}}$ 200P 3K HS n.d.

http://composites.chomarat.com/en/product/c-weave-200p-3k-hs/ (accessed February 7, 2018).

[30] Gividi Fabrics srl. Data sheet and product information. 2016.

[31] Pernice F, Qi G, Senokos E, Anthony DB, Nguyen S, Shirshova N, et al. Effect of material constituents on the tensile and shear properties of carbon fibre $\mathrm{C}$-weave/carbon aerogel based Structural Power supercapacitors. Prep 2019.

[32] De Carvalho NV, Pinho ST, Robinson P. Numerical modelling of woven composites: Biaxial loading. Compos Part A Appl Sci Manuf 2012;43:1326-37.

[33] Tan P, Tong L, Steven GP. Micromechanics models for the elastic constants and failure strengths of plain weave composites. Compos Struct 1999;47:797-804.

[34] Lomov SV, Verpoest I, Cichosz J, Hahn C, Ivanov DS, Verleye B. Meso-level textile composites simulations: Open data exchange and scripting. J Compos Mater 2014;48:621-37.

[35] Wehrkamp-Richter T, De Carvalho NV, Pinho ST. Predicting the non-linear mechanical response of triaxial braided composites. Compos Part A Appl Sci Manuf 2018;114:117-35.

[36] Dassault Systèmes Simulia. Analysis User's Guide (6.14) Volume V. vol. V. 2014.

[37] Cornelissen B, Sachs U, Rietman B, Akkerman R. Dry friction characterisation of carbon fibre tow and satin weave fabric for composite applications. Compos Part A Appl Sci Manuf 2014;56:127-35.

[38] Badel P, Gauthier S, Vidal-Sallé E, Boisse P. Rate constitutive equations for computational analyses of textile composite reinforcement mechanical behaviour during forming. Compos Part A Appl Sci Manuf 2009;40:997-1007.

[39] Mimaroglu A, Unal H, Arda T. Friction and wear performance of pure and glass fibre reinforced poly-ether-imide on polymer and steel counterface materials. Wear 2007;262:1407-13.

[40] Boisse P, Gasser A, Hagege B, Billoet J-L. Analysis of the mechanical behavior of woven fibrous material using virtual tests at the unit cell level. J Mater Sci 2005;40:5955-62.

[41] Lin H, Clifford MJ, Long AC, Sherburn M. Finite element modelling of fabric shear Finite element modelling of fabric shear 2009.

[42] Dassault Systèmes Simulia. Abaqus Benchmarks Guide (6.13). 2013.

[43] Boisse P, Hamila N, Guzman-Maldonado E, Madeo A, Hivet G, dell'Isola F. The biasextension test for the analysis of in-plane shear properties of textile composite reinforcements and prepregs: a review. Int J Mater Form 2017;10:473-92.

[44] De Carvalho N V., Pinho ST, Robinson P. Analytical modelling of the compressive and tensile response of woven composites. Compos Struct 2012;94:2724-35.

[45] Naouar N, Vidal-Salle E, Maire E, Schneider J, Boisse P. Meso F.E. simulation of composite reinforcement deformation based on X-ray computed tomography. Mater Tech 2016;104:16576.

[46] Ali MA, Umer R, Khan KA, Cantwell WJ. In-plane virtual permeability characterization of 3D woven fabrics using a hybrid experimental and numerical approach. Compos Sci Technol 2019;173:99-109.

[47] Pazmino J, Mathieu S, Carvelli V, Boisse P, Lomov S V. Numerical modelling of forming of a non-crimp 3D orthogonal weave E-glass composite reinforcement. Compos Part A Appl Sci Manuf 2015;72:207-18.

[48] Matveev M, Long A, Brown L, Jones I. Effects of layer shift and yarn path variability on mechanical properties of a twill weave composite. J Compos Mater 2017;51:913-25.

[49] Breiling KB, Adams DO, Verhulst MA. Effects of nesting on compression-loaded 2-D woven textile composites. J Compos Mater 1996;30:1710-28.

[50] De Carvalho NV, Pinho ST, Robinson P. An experimental study of failure initiation and propagation in 2D woven composites under compression. Compos Sci Technol 2011;71:131625.

[51] Irizar MO. Quasi-static and fatigue delamination characterisation for carbon fibre reinforced woven laminates: Investigation into the nesting effect between layers. Mondragon Unibertsitatea, 2016.

[52] Dos Santos Felipe RCT, Felipe RNB, De Melo Caldas Batista AC, Aquino EMF. Polymer composites reinforced with hybrid fiber fabrics. Mater Res 2017;20:555-67. 


\section{Figure Captions}

Fig. 1. Surface scans of the fabrics (a) C, $2 \mathrm{~mm}$ scale bar, (b) G, $2 \mathrm{~mm}$ scale bar, (c) out of-plane compression test setup and (d) video gauge image capture, $5 \mathrm{~mm}$ scale bar.

Fig. 2. Optical micrographs of cross-sections of the fabrics (a) C, $1 \mathrm{~mm}$ scale bar, (b) G, $200 \mu \mathrm{m}$ scale bar; fabric monolayer unit cell geometry and fine mesh: (c) C, and (d) G.

Fig. 3. Mesh convergence analysis for compression of $C$ and $G$ fabric monolayers and comparison with experimental ranges.

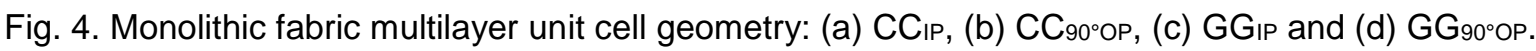

Fig. 5. Hybrid fabric multilayer unit cell geometry: (a) CG, (b) GCG, (c) CGC $C_{\mathbb{P}}$ and (c) CGC $90^{\circ} \mathrm{OP}$.

Fig. 6. Experimental compressibility ranges and model predictions for (a) monolithic stacking sequences ( $\mathrm{CC}_{\mathbb{P}}, \mathrm{CC}_{90^{\circ} \mathrm{OP}}, \mathrm{GG}_{\mathbb{P}}$ and $\mathrm{GG}_{90^{\circ} \mathrm{OP}}$ ) and (b) hybrid stacking sequences (CG, GCG, CGC

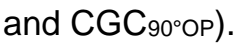

Fig. 7. Local fibre volume fraction distribution: (a) $\mathrm{CC}_{\mathbb{I P}}$ and (b) $\mathrm{CC}_{90^{\circ} \mathrm{OP}}(0.6 \mathrm{MPa})$; (c) $\mathrm{CGC}_{\mathbb{P}}$ and (d) $\mathrm{CGC}_{90^{\circ} \mathrm{OP}}(0.3 \mathrm{MPa})$.

Fig. 8. Three-point perspective projection of (a) volume reconstruction and (b) orthogonal slices (midplane: $x y, x z, y z$ ) of a sample extracted from a $\mu C T$ scan of a CGC/epoxy composite panel specimen, containing four complete hUCs; (c) optical micrograph of a CGC/epoxy composite panel cross-section, containing one representative hUC, $500 \mu \mathrm{m}$ scale bar.

Fig. 9. Three-point perspective (above) and third-angle multi-view orthographic (below) projections of orthogonal slices (midplane $x y, x z, y z$ ) extracted from the CGC/epoxy $\mu$ CT scan, displaying one representative hUC from each sample: (a), (b) and (c); composite images produced by combining two identical images with different greyscale colormap limits to enhance contrast in carbon plies.

Fig. 10. Predicted laminate midplane cuts through (a) $\mathrm{CGC}_{1 \mathrm{P}}$, (b) $\mathrm{CGC}_{90^{\circ} \mathrm{OP}}$ and (c) $\mathrm{CGC}_{36^{\circ} \mathrm{OP}}$ models; (d) segmented laminate midplane slice from the CGC/epoxy $\mu \mathrm{CT}$ scan, containing one representative hUC (G/epoxy in teal, C/epoxy in beige); Predicted cross-sections through (e) $\mathrm{CGC}_{\mathbb{P}}$, (f) $\mathrm{CGC}_{90^{\circ} \mathrm{OP}}$ and (g) $\mathrm{CGC}_{36^{\circ} \mathrm{OP}}$ models; (h) segmented optical micrograph of a CGC/epoxy composite panel crosssection, containing one representative hUC.

\section{Table Captions}

Table 1. Fabric specifications.

Table 2. Mean yarn geometric parameters of undeformed fabric; values in parentheses indicate one standard deviation.

Table 3. Yarn constitutive model parameters.

Table 4. Non-linear transverse stiffness coefficients.

Table 5. Modified geometric parameters in hUC models. 


\section{Figures}

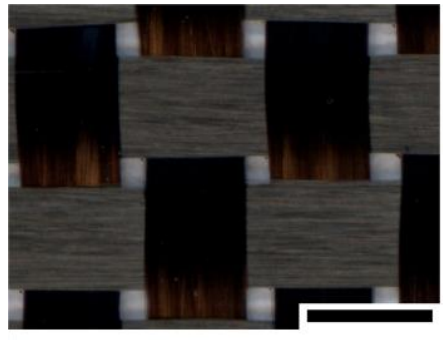

(a)

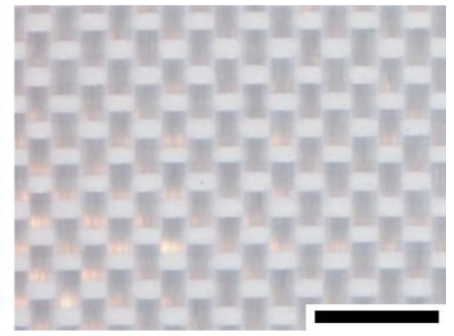

(b)

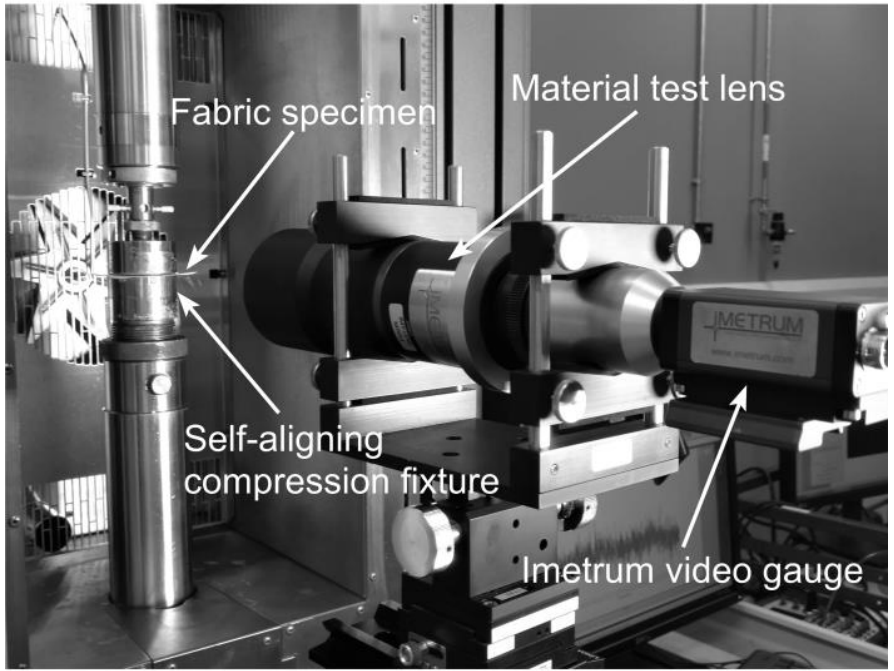

(c)

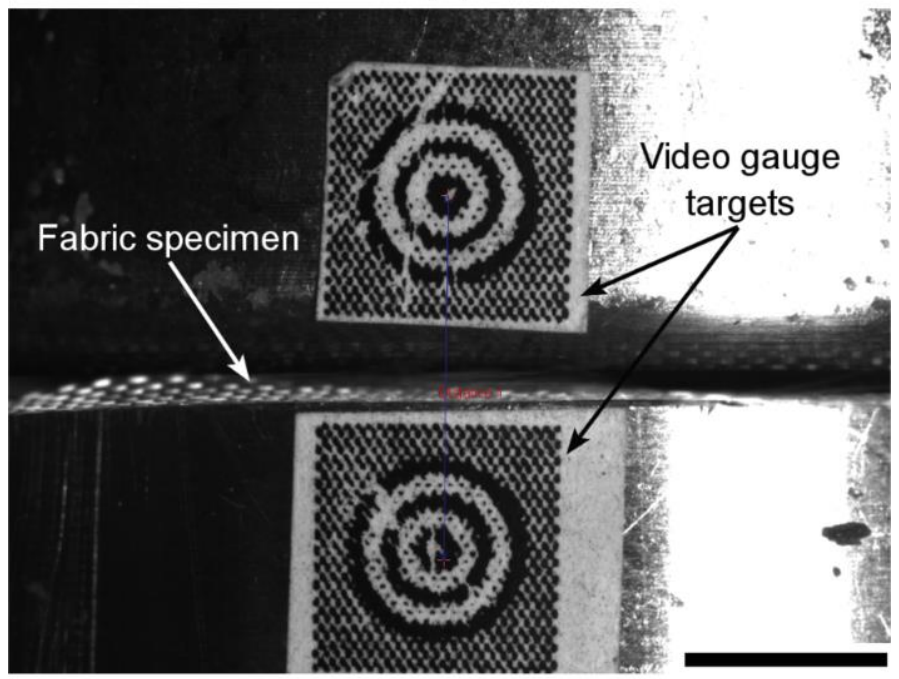

(d)

Fig. 1. Surface scans of the fabrics (a) C, $2 \mathrm{~mm}$ scale bar, (b) G, $2 \mathrm{~mm}$ scale bar, (c) out of-plane compression test setup and (d) video gauge image capture, $5 \mathrm{~mm}$ scale bar. 


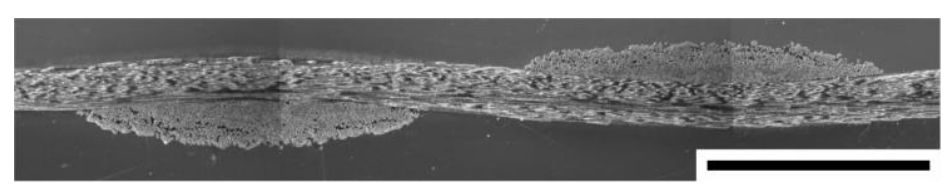

(a)

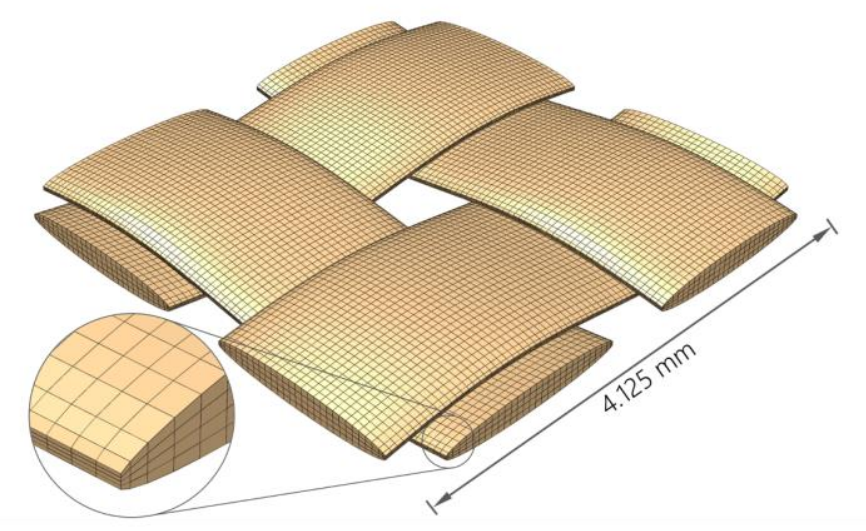

(c)

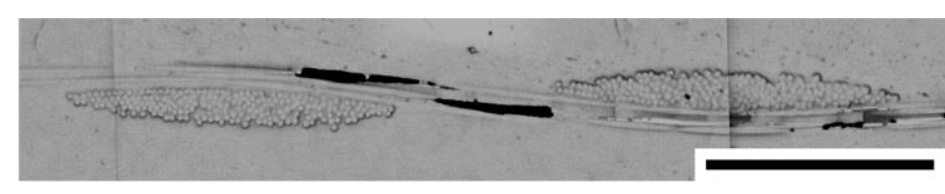

(b)

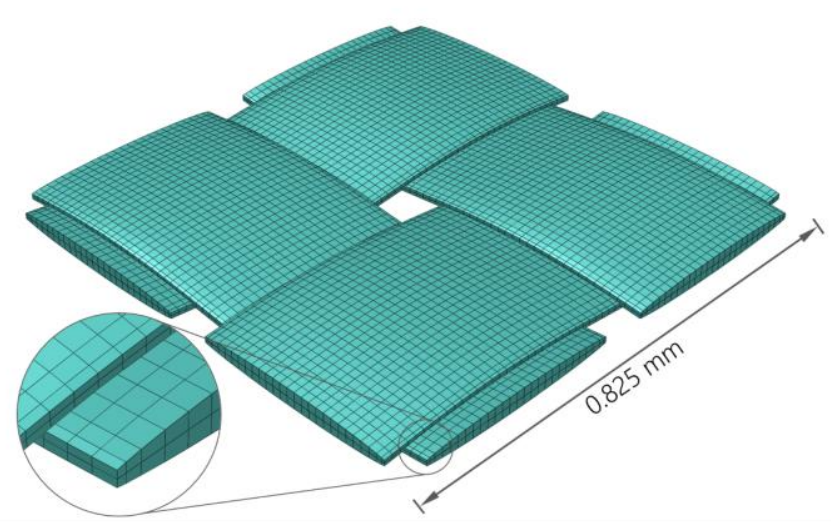

(d)

Fig. 2. Optical micrographs of cross-sections of the fabrics (a) C, $1 \mathrm{~mm}$ scale bar, (b) G, $200 \mu \mathrm{m}$ scale bar; fabric monolayer unit cell geometry and fine mesh: (c) C, and (d) G. (Colour print)

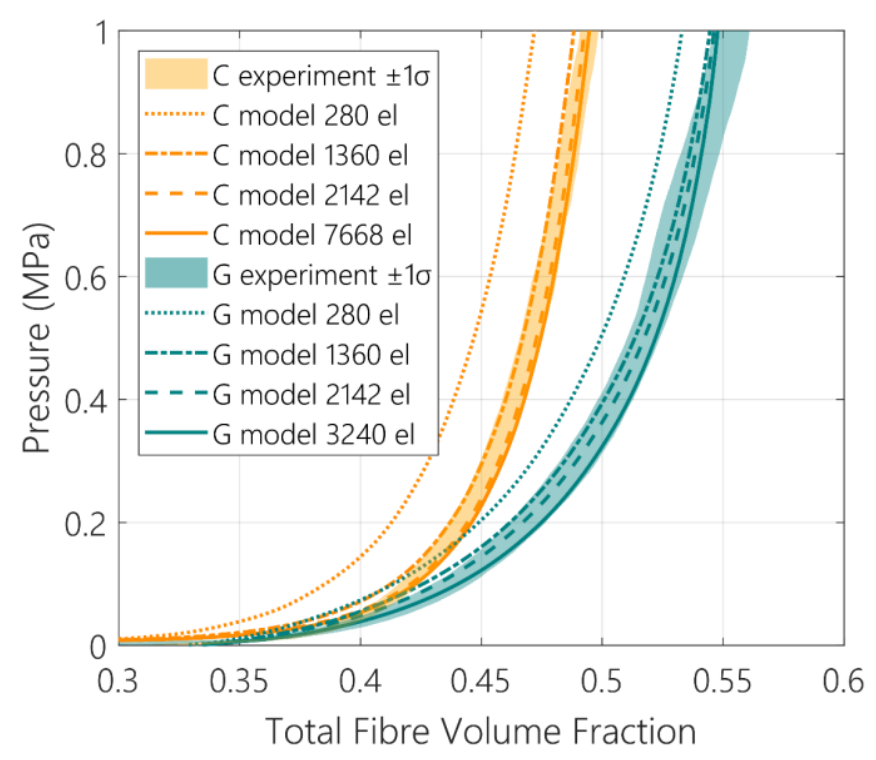

Fig. 3. Mesh convergence analysis for compression of $C$ and $G$ fabric monolayers and comparison with experimental ranges. (Colour print) 


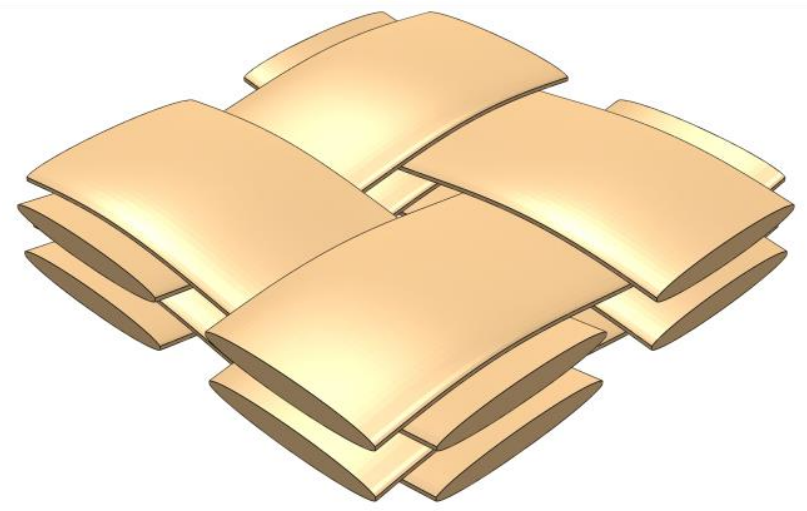

(a)

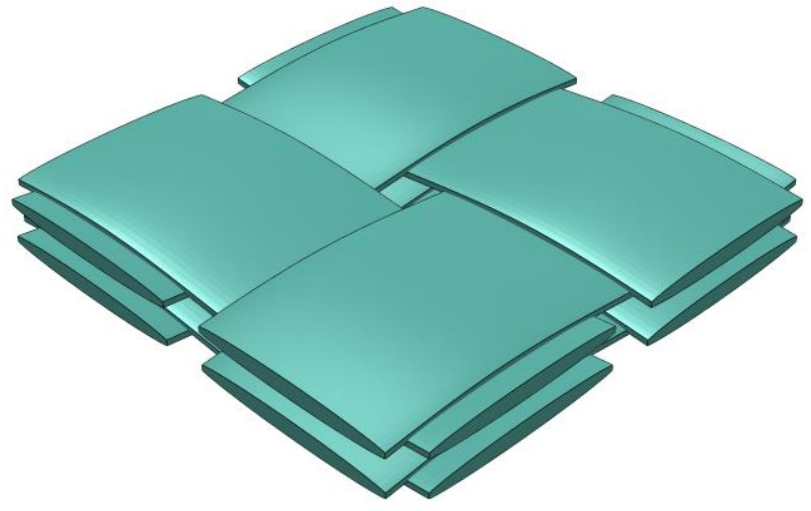

(c)

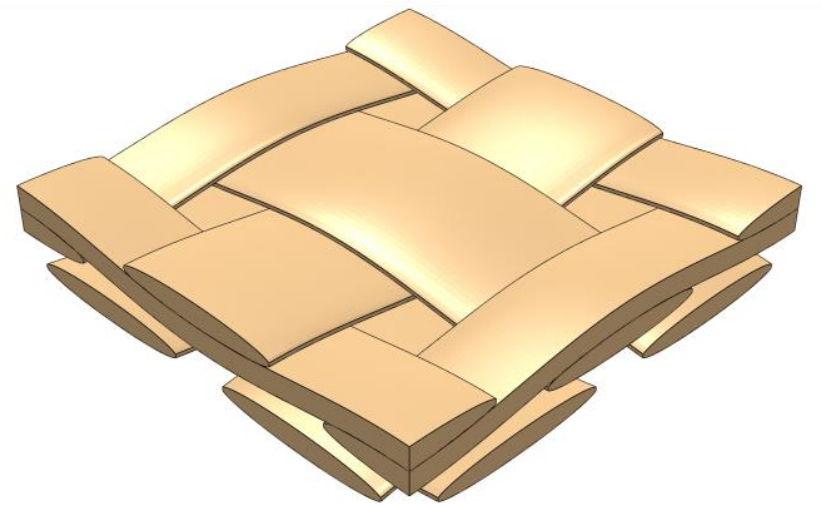

(b)

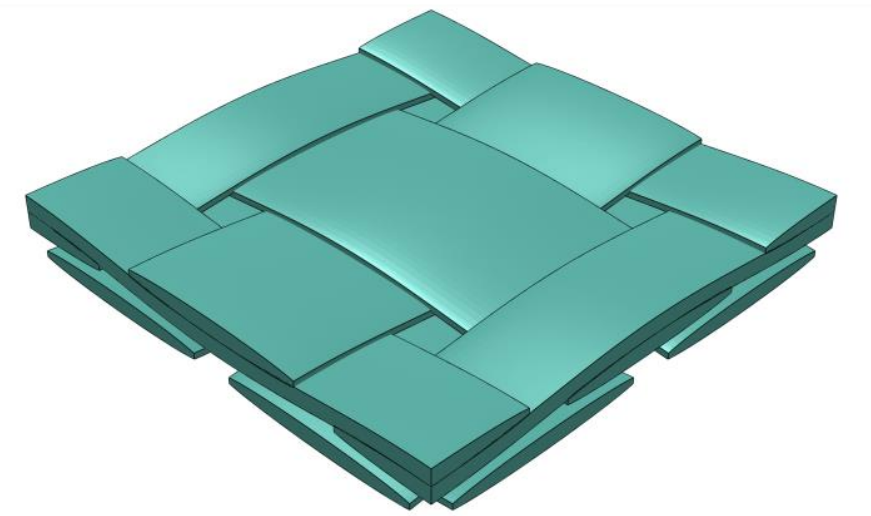

(d)

Fig. 4. Monolithic fabric multilayer unit cell geometry: (a) $\mathrm{CC}_{\mathbb{I P}}$, (b) $\mathrm{CC}_{90^{\circ} \mathrm{OP}}$, (c) $\mathrm{GG}_{\mathrm{IP}}$ and (d) $\mathrm{GG}_{90^{\circ} \mathrm{OP}}$. (Colour print) 


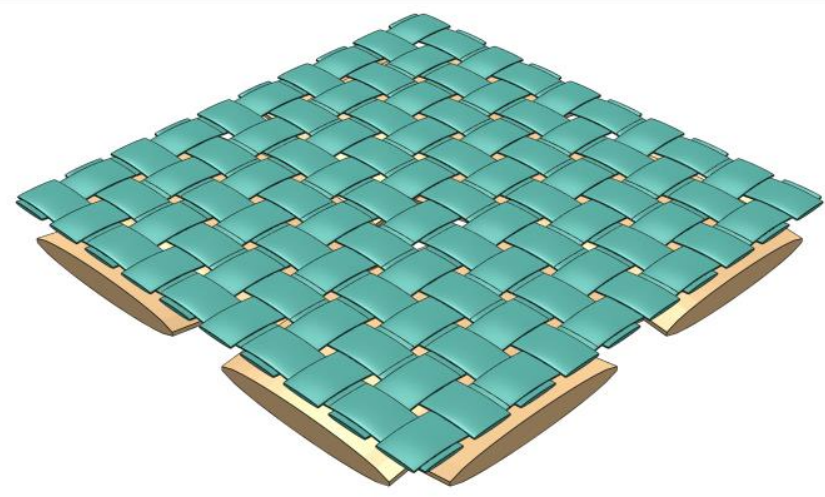

(a)

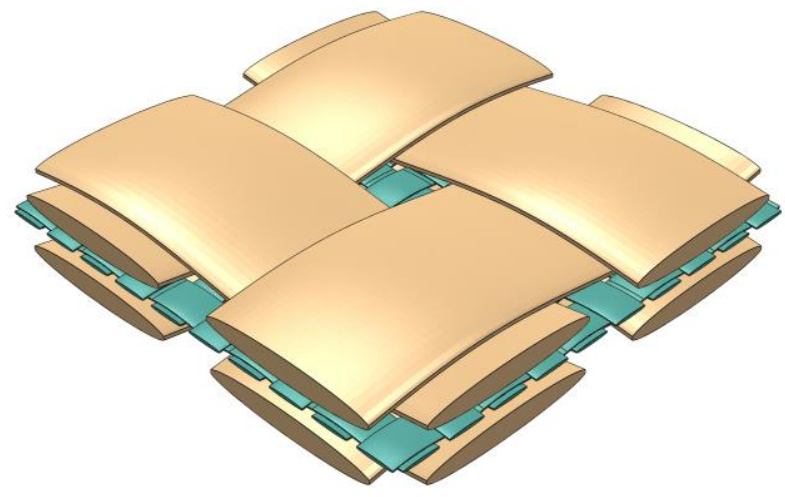

(c)

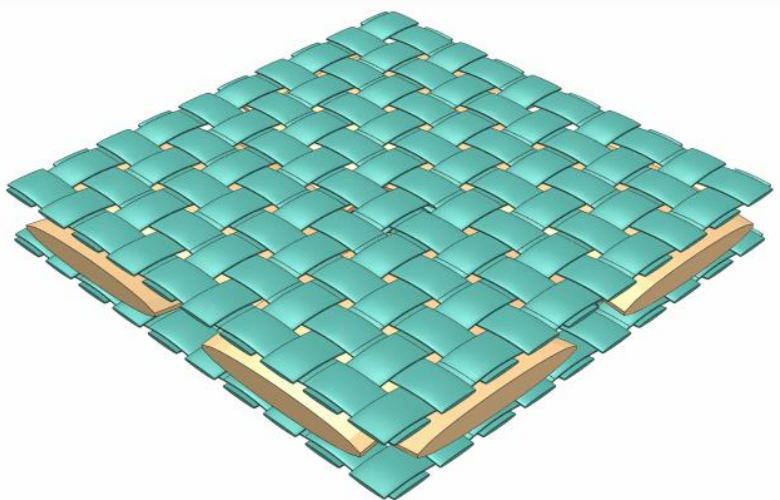

(b)

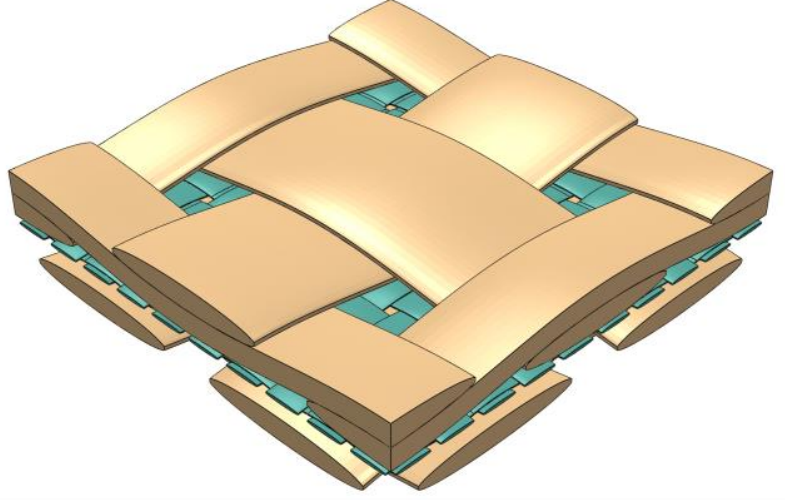

(d)

Fig. 5. Hybrid fabric multilayer unit cell geometry: (a) CG, (b) GCG, (c) $C G C_{\mathbb{P}}$ and (c) CGC90०0P. (Colour print)

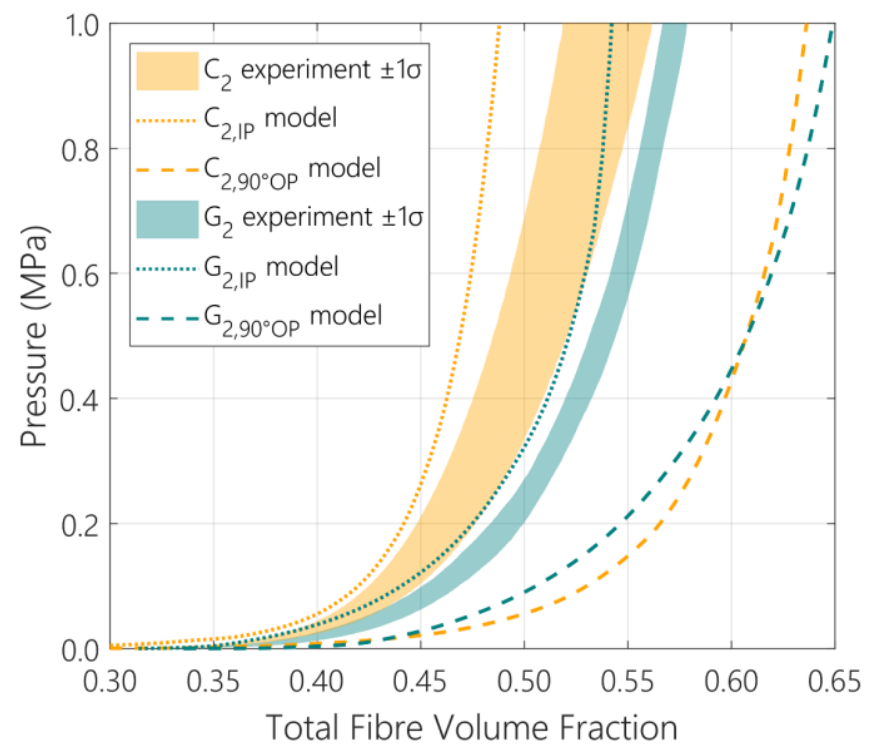

(a)

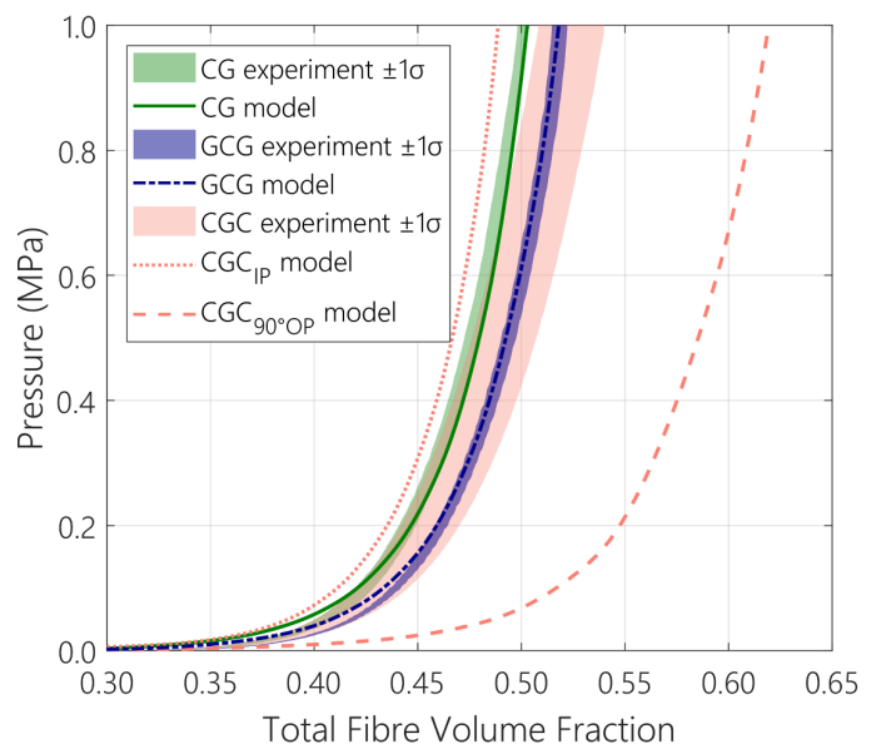

(b)

Fig. 6. Experimental compressibility ranges and model predictions for (a) monolithic stacking sequences ( $\mathrm{CC}_{\mathbb{I P}}, \mathrm{CC}_{90^{\circ} \mathrm{OP}}, \mathrm{GG}_{\mathbb{P}}$ and $\mathrm{GG}_{90^{\circ} \mathrm{OP}}$ ) and (b) hybrid stacking sequences (CG, GCG, CGC

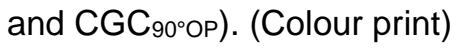



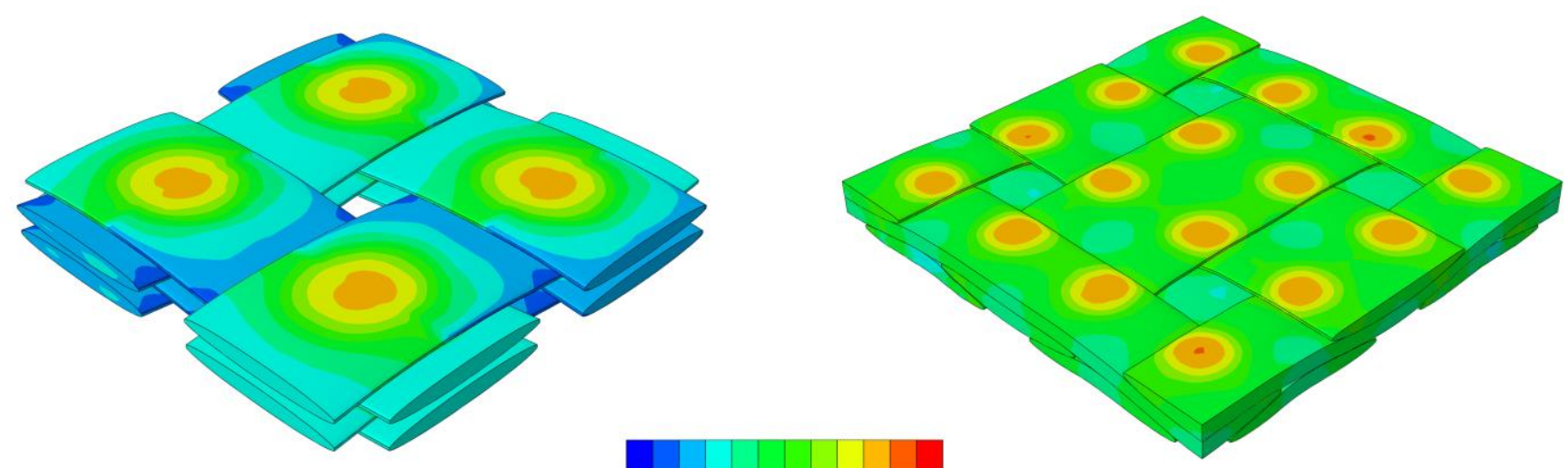

(a)

\% 47505357606366697276798285

(b)

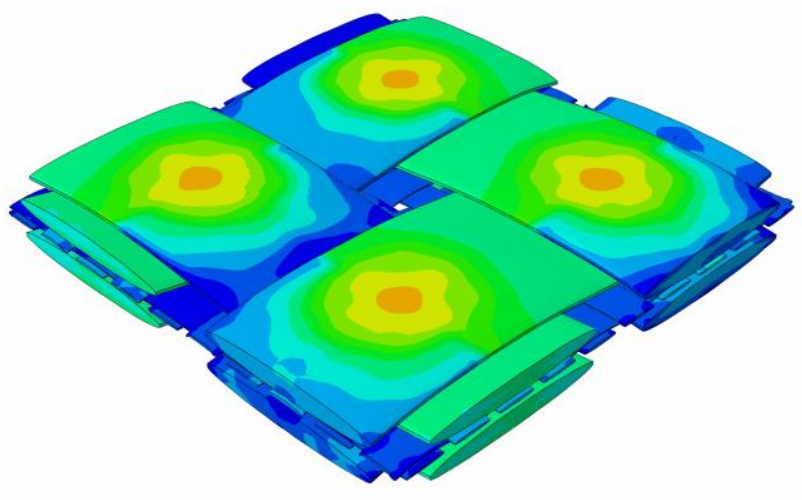

(c)

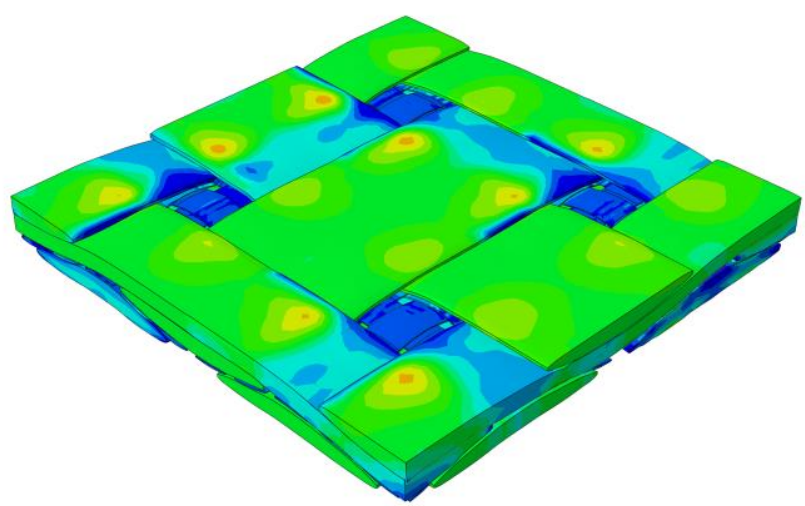

(d)

Fig. 7. Local fibre volume fraction distribution: (a) $\mathrm{CC}_{\mathbb{P}}$ and (b) $\mathrm{CC}_{90^{\circ} \mathrm{OP}}(0.6 \mathrm{MPa})$; (c) $\mathrm{CGC}_{\mathbb{P}}$ and (d) $\mathrm{CGC}_{90^{\circ} \mathrm{OP}}(0.3 \mathrm{MPa})$. (Colour print) 


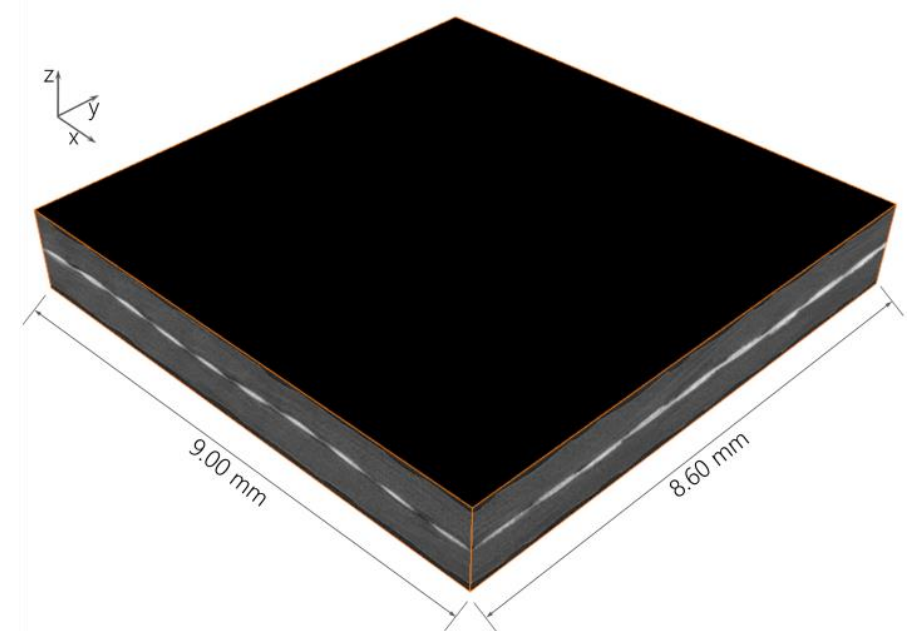

(a)

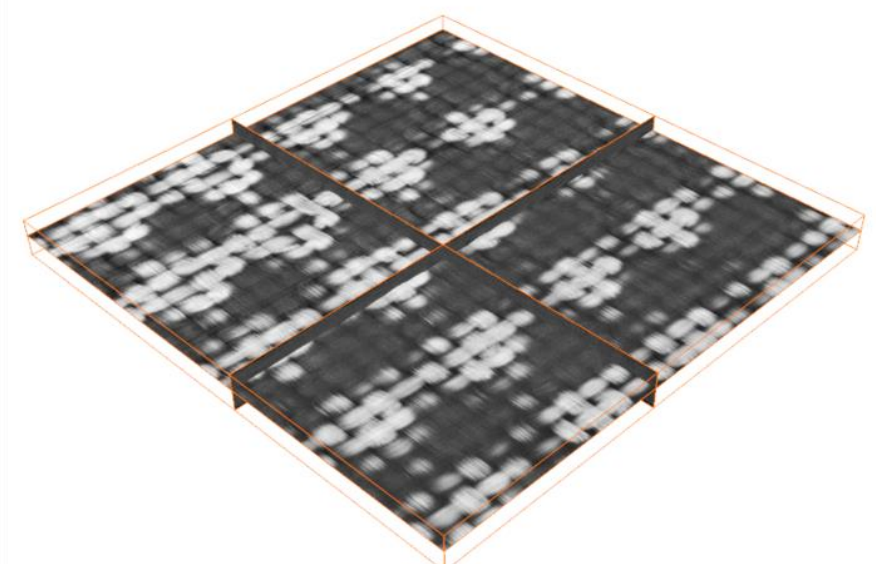

(b)

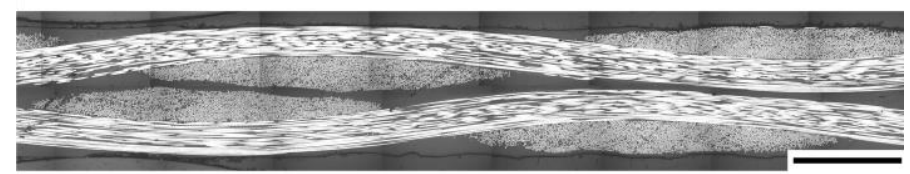

(c)

Fig. 8. Three-point perspective projection of (a) volume reconstruction and (b) orthogonal slices (midplane: $x y, x z, y z$ ) of a sample extracted from a $\mu C T$ scan of a CGC/epoxy composite panel specimen, containing four complete hUCs; (c) optical micrograph of a CGC/epoxy composite panel cross-section, containing one representative hUC, $500 \mu \mathrm{m}$ scale bar. (Colour print) 

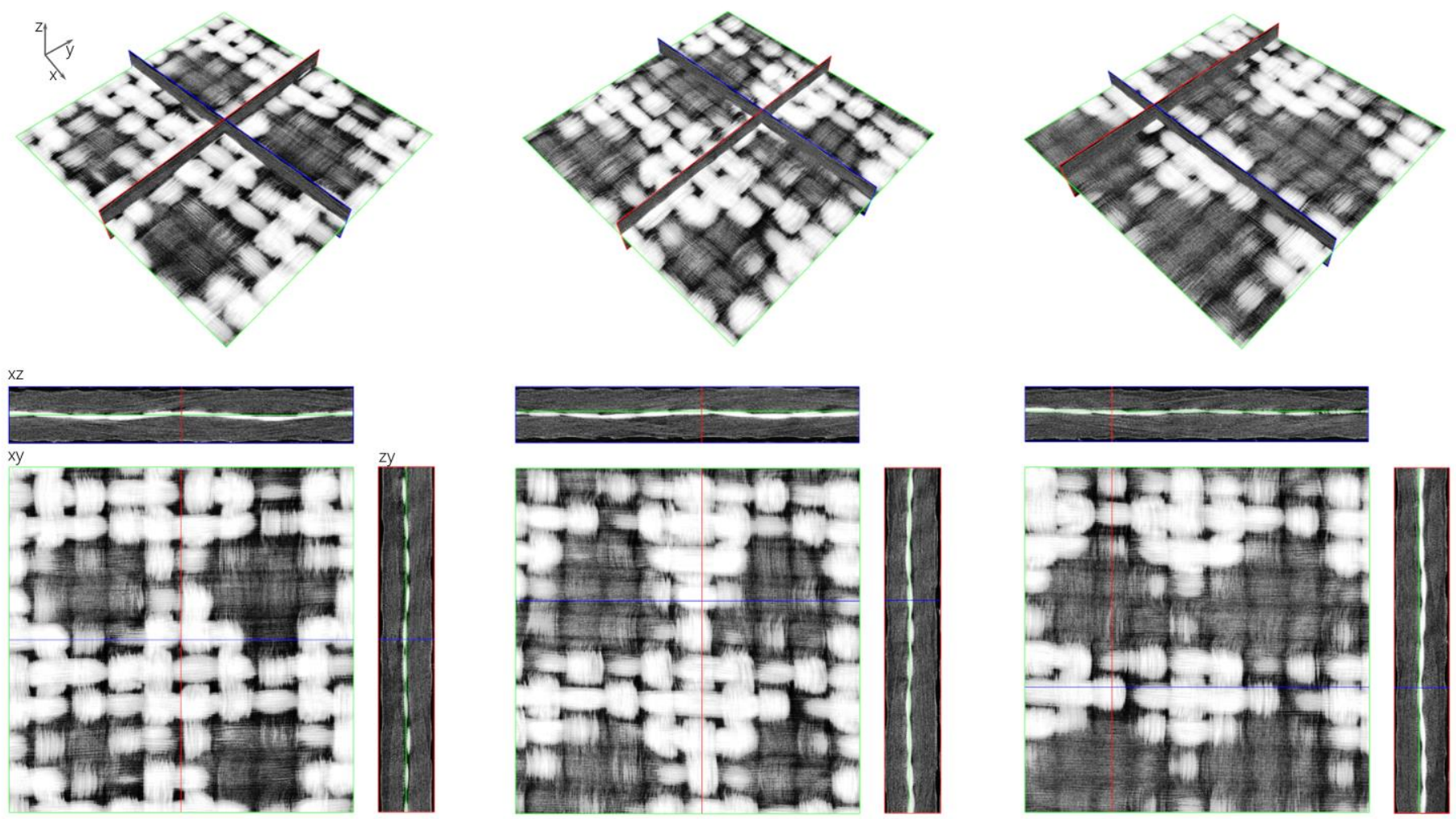

(a)

(b)

(c)

Fig. 9. Three-point perspective (above) and third-angle multi-view orthographic (below) projections of orthogonal slices (midplane $x y, x z$, zy) extracted from the CGC/epoxy $\mu$ CT scan, displaying one representative hUC from each sample: (a), (b) and (c); composite images produced by combining two identical images with different greyscale colormap limits to enhance contrast in carbon plies. (Colour print) 


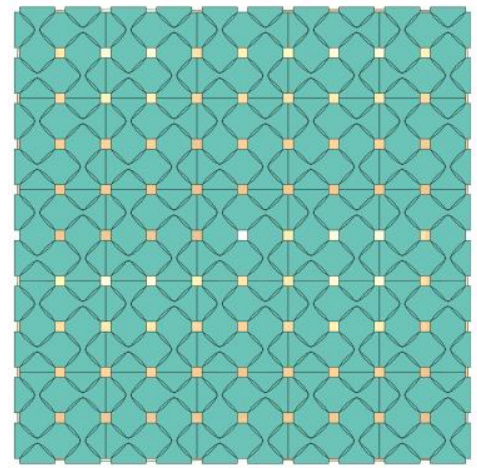

(a)

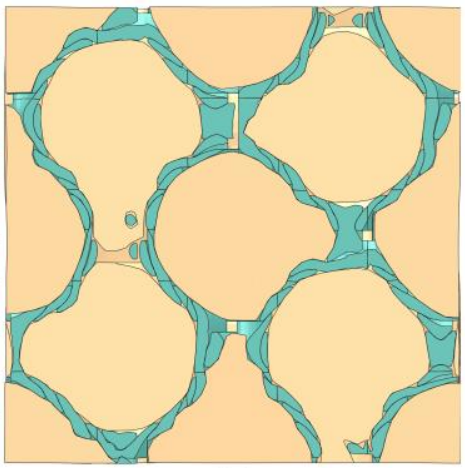

(b)

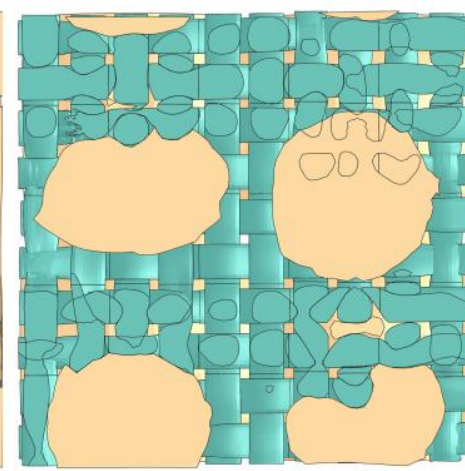

(c)

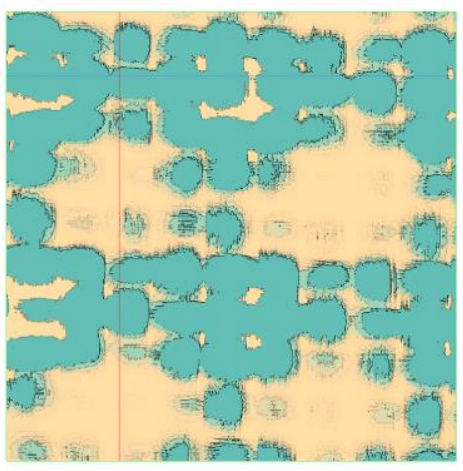

(d) (e)

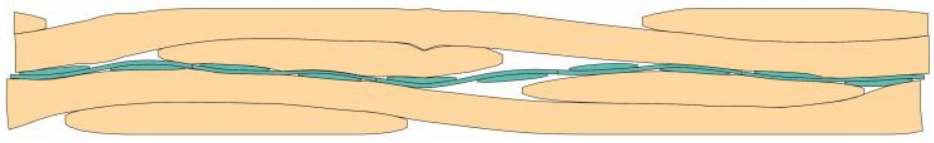

(g)

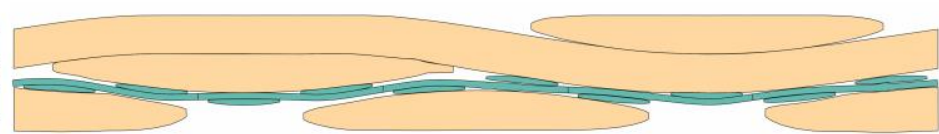

(f)

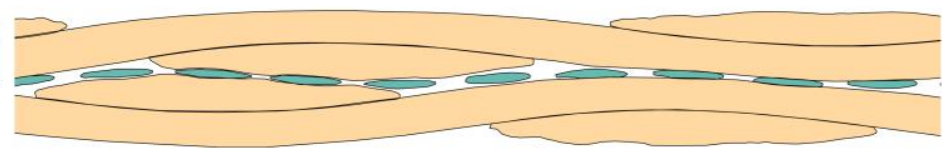

(h)

Fig. 10. Predicted laminate midplane cuts through (a) $\mathrm{CGC}_{1 \mathrm{P}}$, (b) $\mathrm{CG}_{90^{\circ} \mathrm{OP}}$ and (c) $\mathrm{CGC}_{36^{\circ} \mathrm{OP}}$ models; (d) segmented laminate midplane slice extracted from the CGC/epoxy $\mu C T$ scan, containing one representative hUC (G/epoxy in teal, C/epoxy in beige); Predicted cross-sections through (e) CGCIP, (f) $\mathrm{CGC}_{90^{\circ} \mathrm{OP}}$ and (g) $\mathrm{CGC}_{36^{\circ} \mathrm{OP}}$ models; (h) segmented optical micrograph of a CGC/epoxy composite panel cross-section, containing one representative hUC. (Colour print) 
Tables

Table 1. Fabric specifications.

\begin{tabular}{ccccccc}
\hline Fabric & Pattern & $\begin{array}{c}\text { Areal density } \\
\left(\mathrm{g} / \mathrm{m}^{2}\right)\end{array}$ & Fibre type & $\begin{array}{c}\text { Fibre tensile modulus } \\
(\mathrm{GPa})\end{array}$ & $\begin{array}{c}\text { Fibre density } \\
\left(\mathrm{g} / \mathrm{cm}^{3}\right)\end{array}$ & Filaments/yarn \\
\hline Carbon, C & Plain & 200 & FT300B (PAN) & 230 & 1.75 & 3000 \\
Glass, G & Plain & 53 & E glass & 71 & 2.55 & 200 \\
\hline
\end{tabular}

Table 2. Mean yarn geometric parameters of undeformed fabric; values in parentheses indicate one standard deviation.

\begin{tabular}{lcccc} 
Fabric & $\begin{array}{c}\text { Width } \\
(\mathrm{mm})\end{array}$ & $\begin{array}{c}\text { Height } \\
(\mathrm{mm})\end{array}$ & $\begin{array}{c}\text { Pitch } \\
(\mathrm{mm})\end{array}$ & $\begin{array}{c}\text { Intra-yarn fibre volume fraction } \\
(\%)\end{array}$ \\
\hline Carbon, C & $1.557( \pm 0.047)$ & $0.200( \pm 0.009)$ & $2.018( \pm 0.087)$ & 46.9 \\
Glass, G & $0.347( \pm 0.056)$ & $0.028( \pm 0.004)$ & $0.419( \pm 0.019)$ & 49.7 \\
\hline
\end{tabular}

Table 3. Yarn constitutive model parameters.

\begin{tabular}{ccccc}
\hline$E_{1}$ & $E_{2}$ & $G_{12}$ & $v_{12}$ & $v_{23}$ \\
\hline$V_{f} E_{f}$ & $a \exp \left(b V_{f}\right)+c \exp \left(d V_{f}\right)$ & $1 \mathrm{MPa}$ & 0 & 0 \\
{$[13,41]$} & {$[12,15,16]$} & {$[38,53]$} & {$[7,16,40,53]$} & {$[7,16,40,53]$} \\
\hline
\end{tabular}

Table 4. Non-linear transverse stiffness coefficients.

\begin{tabular}{ccccc}
\hline Fabric & $a$ & $b$ & $c$ & $d$ \\
\hline Carbon, C & 0.45 & 23.14 & $2.4 \mathrm{e}-15$ & 61.20 \\
Glass, G & 187.76 & 14.09 & $3.9 \mathrm{e}-16$ & 65.60 \\
\hline
\end{tabular}

Table 5. Modified geometric parameters in hUC models.

\begin{tabular}{ccc}
\hline Fabric & Width $(\mathrm{mm})$ & Pitch $(\mathrm{mm})$ \\
\hline Carbon, $\mathrm{C}$ & 1.5500 & 2.0625 \\
Glass, $\mathrm{G}$ & 0.3395 & 0.4125 \\
\hline
\end{tabular}

